\title{
Computing the Full Non-Rigid Group of Trimethylborane and Cyclohaxane Using Wreath Product
}

\author{
Enoch Suleiman ${ }^{1 *}$, M. S. Audu ${ }^{2}$ \\ ${ }^{1}$ Department of Mathematics, Federal University Gashua, Gashua, Nigeria \\ ${ }^{2}$ Department of Mathematics, University of Jos, Jos, Nigeria \\ Email: *enochsuleiman@gmail.com, audumso2@gmail.com
}

How to cite this paper: Suleiman, E. and Audu, M.S. (2020) Computing the Full Non-Rigid Group of Trimethylborane and Cyclohaxane Using Wreath Product. American Journal of Computational Mathematics, 10, 23-30.

https://doi.org/10.4236/ajcm.2020.101002

Received: December 11, 2019

Accepted: January 6, 2020

Published: January 9, 2020

Copyright (c) 2020 by author(s) and Scientific Research Publishing Inc.

This work is licensed under the Creative Commons Attribution International License (CC BY 4.0).

http://creativecommons.org/licenses/by/4.0/

\begin{abstract}
This paper computes the group and character table of Trimethylborane and Cyclohaxane. Results show that the groups are isomorphic to the wreath products $C_{3} w r C_{2}$ and $C_{2} w r C_{6}$ with orders 81 and 384 and with 17 and 28 conjugacy classes respectively, where $C_{n}$ denotes a cyclic group of order $n$.
\end{abstract}

\section{Keywords}

Non-Rigid Molecule Group, Trimethylborane, Cyclohaxane, Wreath Product, Character Table, Conjugacy Classes

\section{Introduction}

Symmetry is very important in group theory. The symmetry of non-rigid molecules is a new field in chemistry. Balasubramanian [1] discussed the importance of finite groups which characterize the algebra of the symmetry of discrete structure as useful in predicting many physical and chemical properties. He further reviewed the formulation of symmetry groups of non-rigid molecules as generalized wreath product groups. He formulated the non-rigid hydrazine molecule $\left(\mathrm{N}_{2} \mathrm{H}_{2}\right)$. He showed that the molecule is non-rigid in that twisting and inversion operations interconvert all the 16 possible conformations into one another. Considering the permutational subgroup of this molecule, he found out that the permutations operations of the non-rigid molecule can be generated by a group product of much simpler groups, known as wreath product and thus it is a group of order 8 . Balasubramanian also used the generating function methods to find the irreducible representation of Boron trimethyl $\mathrm{B}\left(\mathrm{CH}_{3}\right)_{3}$ and also the 
non-rigid Deuterion spin species of $\mathrm{B}\left(\mathrm{CD}_{3}\right)_{3}$ and also that of Butane. Hamadanian and Ashrafi [2] studied the full NRG of trimethylamine $\mathrm{N}\left(\mathrm{CH}_{3}\right)_{3}$ and prove that is a group of order 1296 with 28 conjugacy classes. Darafsher et al. [3] calculated the irreducible representations and character table of Tetramethylene molecule. They first specify the algebraic structure of the f-NRG of tetramethylethylene molecule with a geometric consideration of dynamic symmetries of the molecule and showed that the $\mathrm{f}-\mathrm{NRG}$ of the molecule can be specified as $\left(\mathbb{Z}_{3}\right) w r\left(\mathbb{Z}_{2} \times \mathbb{Z}_{2}\right)$. Then using the package GAP, the $\mathrm{f}-\mathrm{NRG}$ group was computed and is shown to be of order 324 permutations. It is a group of 45 conjugacy classes of elements and contains exactly 45 irreducible characters. Darafsher et al. [4] studied the f-NRG of tetranitrocubane and octanitrocubane and prove that these are groups of order 384 and 12288 respectively. The f-NRG group of tetranitrocubane was shown to be isomorphic to $\mathbb{Z}_{2} w r S_{4}$ while that of octanitrocubane was shown to be isomorphic to $\left(\mathbb{Z}_{2}\right) w r\left(S_{4} \times \mathbb{Z}_{2}\right)$. The conjugacy classes and the character tables of the two molecules were computed using the GAP package.

Darafsher et al. [5] studied the 2,3-dimethylbutane and found the structure of the full non-rigid group of this molecule to be isomorphic to the semidirect product of four copies of the cyclic group $\mathbb{Z}_{3}$ by the cyclic group $\mathbb{Z}_{2}$, that is, $\left(\mathbb{Z}_{3} \times \mathbb{Z}_{3} \times \mathbb{Z}_{3} \times \mathbb{Z}_{3}\right) \rtimes \mathbb{Z}_{2}$, and the complex character table of the group was computed by the package GAP. Moghani et al. [6] studied the non-rigid group of 2,4-dimethylbenzene that is isomorphic to $\mathbb{Z}_{3} \times\left(\mathbb{Z}_{3} w r \mathbb{Z}_{2}\right)$ with order 36. It was shown that the group has 12 dominant classes and the Markaracter table, the table of all integer-valued characters and the unit subduced cycle index Table of the full non-rigid group of 2,4-dimethyl benzene are derived for the first time. Moghadam et al. [7] computed the f-NRG of fluoro(dimethyl)borane and (difluoromethyl)borane with $C_{s}$ point group. They proved that the f-NRG of the two molecules is of order 18 and 6 with 6 and 3 conjugacy classes respectively. The conjugacy classes and the Character tables were computed using the GAP package. Moghadam et al. [8] studied the symmetry of non-rigid Cis- and trans-dimethyl diborane, trimethyl diborane and tetramethyl diborane with different point groups and the conjugacy classes and the irreducible character tables of the molecules are also calculated.

The character table which is being needed for the classification of wave functions, determination of selection rules, etc. in which were computed for Trimethylborane and Cyclohaxanethe groups are found to be isomorphic to the wreath products $C_{3} w r C_{2}$ and $C_{2} w r C_{6}$ with orders 81 and 384 and with 17 and 28 conjugacy classes respectively. We use the GAP package to get the required results.

The next section shows the results that are obtained and some basic definitions while the following section gives the conclusion.

\section{Results and Computation}

Let $\Omega$ be an arbitrary set; we shall often refer to its elements as points. A bijective 
(a one-to-one, onto mapping) of $\Omega$ onto itself is called a permutation of $\Omega$. The set of all permutations of $\Omega$ forms a group under composition of mappings, called the Symmetric group of $\Omega$. We shall denote this group by $\operatorname{Sym}(\Omega)$ (or $S_{\Omega}$ ), and write $S_{n}$ to denote the special group $\operatorname{Sym}(\Omega)$ when $n$ is a positive integer and $\Omega=\{1,2, \cdots, n\}$. A permutation group is just a subgroup of Symmetric group. If $\Gamma$ and $\Delta$ are nonempty sets, then we call $\Gamma^{\Delta}$ to denote the set of all functions from $\Delta$ to $\Gamma$. In the case that $C$ is a group, we turn $C^{\Delta}$ into a group by defining product "pointwise"

$$
f g(\gamma):=f(\gamma) g(\gamma)
$$

for all $f, g \in C^{\Delta}$ and $\gamma \in \Delta$ where the product in the right is in $C$.

Let $C$ and $D$ be groups and suppose $D$ acts on the nonempty set $\Delta$. Then the wreath product of $C$ by $D$ is defined with respect to this action is defined to be the semidirect product $C^{\Delta} \rtimes D=C w r D$ where $D$ acts on the group $C^{\Delta}$ via

$$
f^{d}(\gamma):=f\left(\gamma^{d^{-1}}\right)
$$

for all $f \in C^{\Delta}, \gamma \in \Delta$ and $d \in D$ and multiplication for all $\left(f_{1}, d_{1}\right),\left(f_{2}, d_{2}\right) \in C w r D$ is given by

$$
\left(f_{1}, d_{1}\right)\left(f_{2}, d_{2}\right)=\left(f_{1} f_{2}^{d_{1}^{-1}}, d_{1} d_{2}\right)
$$

(See [9])

\section{1. f-NRG of Trimethylborane}

Consider the trimethylborane compound $\mathrm{B}\left(\mathrm{CH}_{3}\right)_{3}$ with the structure.

The speediness of the rotation of the methyl group is considered appropriately high that makes the mean time dynamical symmetry of the molecules makes sense. First considering the symmetry of $\mathrm{CH}_{3}$ which is a cyclic group of order 3 namely $\mathbb{Z}_{3}$ denoted by $A:=\langle(1,2,3)\rangle$ (as shown in Figure 1 ). Also the remaining 3 corners of the framework are given by: $B_{1}:=\langle(4,5,6)\rangle$, $B_{2}:=\langle(7,8,9)\rangle, \quad B_{3}:=\langle(10,11,12)\rangle$ where $B_{i}$ is the symmetry of the $\mathrm{CH}_{3}$ whose carbon atom is marked as $i, 1 \leq i \leq 3$. Therefore the full symmetry of trimethylborane is: $G=\left(B_{1} \times B_{2} \times B_{3}\right) \rtimes A$. Which we can write in terms of wreath product as $G:=\mathbb{Z}_{3} w r \mathbb{Z}_{3}$. We used GAP package to get the group as follows:

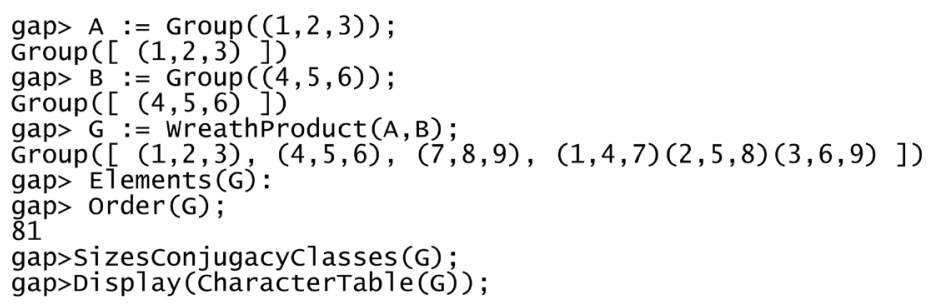

(See [10])

From the foregoing Table 1, The Representative of conjugacy classes of the group and Table 2, The Character table obtained gives room for the needed classification of wavefunctions, determination of selection rules, and so on. 
Table 1. The representative of conjugacy classes of the group.

\begin{tabular}{|c|c|c|c|}
\hline $\mathrm{S} / \mathrm{N}$ & Representatives & Size & Name \\
\hline 1. & () & 1 & $1 \mathrm{a}$ \\
\hline 2. & $(7,8,9)$ & 3 & $3 a$ \\
\hline 3. & $(7,9,8)$ & 3 & $3 b$ \\
\hline 4. & $(4,5,6)(7,8,9)$ & 3 & $3 c$ \\
\hline 5. & $(4,5,6)(7,9,8)$ & 3 & $3 \mathrm{~d}$ \\
\hline 6. & $(4,6,5)(7,8,9)$ & 3 & $3 e$ \\
\hline 7. & $(4,6,5)(7,9,8)$ & 3 & $3 f$ \\
\hline 8. & $(1,4,7)(2,5,8)(3,6,9)$ & 9 & $3 g$ \\
\hline 9. & $(1,4,7,2,5,8,3,6,9)$ & 9 & $3 \mathrm{~h}$ \\
\hline 10. & $(1,4,7,3,6,9,2,5,8)$ & 9 & $3 \mathrm{i}$ \\
\hline 11. & $(1,7,4)(2,8,5)(3,9,6)$ & 9 & $3 j$ \\
\hline 12. & $(1,4,7)(2,5,8)(3,6,9)$ & 9 & $3 \mathrm{k}$ \\
\hline 13. & $(1,4,7,2,5,8,3,6,9)$ & 9 & $9 \mathrm{a}$ \\
\hline 14. & $(1,4,7,3,6,9,2,5,8)$ & 9 & $9 b$ \\
\hline 15. & $(1,7,4)(2,8,5)(3,9,6)$ & 9 & 31 \\
\hline 16. & $(1,7,5,2,8,6,3,9,4)$ & 9 & $9 c$ \\
\hline 17. & $(1,7,6,3,9,5,2,8,4)$ & 9 & $9 \mathrm{~d}$ \\
\hline
\end{tabular}

Table 2. Character Table for Trimethylborane.

\begin{tabular}{cccccccccccccccccc}
\hline & $1 \mathrm{a}$ & $3 \mathrm{a}$ & $3 \mathrm{~b}$ & $3 \mathrm{c}$ & $3 \mathrm{~d}$ & $3 \mathrm{e}$ & $3 \mathrm{f}$ & $3 \mathrm{~g}$ & $3 \mathrm{~h}$ & $3 \mathrm{i}$ & $3 \mathrm{j}$ & $3 \mathrm{k}$ & $9 \mathrm{a}$ & $9 \mathrm{~b}$ & $3 \mathrm{l}$ & $9 \mathrm{c}$ & $9 \mathrm{~d}$ \\
\hline $2 \mathrm{P}$ & $1 \mathrm{a}$ & $3 \mathrm{~b}$ & $3 \mathrm{a}$ & $3 \mathrm{f}$ & $3 \mathrm{e}$ & $3 \mathrm{~d}$ & $3 \mathrm{c}$ & $3 \mathrm{j}$ & $3 \mathrm{i}$ & $3 \mathrm{~h}$ & $3 \mathrm{~g}$ & $3 \mathrm{l}$ & $9 \mathrm{~d}$ & $9 \mathrm{c}$ & $3 \mathrm{k}$ & $9 \mathrm{~b}$ & $9 \mathrm{a}$ \\
$3 \mathrm{P}$ & $1 \mathrm{a}$ & $1 \mathrm{a}$ & $1 \mathrm{a}$ & $1 \mathrm{a}$ & $1 \mathrm{a}$ & $1 \mathrm{a}$ & $1 \mathrm{a}$ & $1 \mathrm{a}$ & $1 \mathrm{a}$ & $1 \mathrm{a}$ & $1 \mathrm{a}$ & $1 \mathrm{a}$ & $3 \mathrm{~g}$ & $3 \mathrm{j}$ & $1 \mathrm{a}$ & $3 \mathrm{~g}$ & $3 \mathrm{j}$ \\
$\mathrm{5P}$ & $1 \mathrm{a}$ & $3 \mathrm{~b}$ & $3 \mathrm{a}$ & $3 \mathrm{f}$ & $3 \mathrm{e}$ & $3 \mathrm{~d}$ & $3 \mathrm{c}$ & $3 \mathrm{j}$ & $3 \mathrm{i}$ & $3 \mathrm{~h}$ & $3 \mathrm{~g}$ & $3 \mathrm{l}$ & $9 \mathrm{~d}$ & $9 \mathrm{c}$ & $3 \mathrm{k}$ & $9 \mathrm{~b}$ & $9 \mathrm{a}$ \\
$7 \mathrm{P}$ & $1 \mathrm{a}$ & $3 \mathrm{a}$ & $3 \mathrm{~b}$ & $3 \mathrm{c}$ & $3 \mathrm{~d}$ & $3 \mathrm{e}$ & $3 \mathrm{f}$ & $3 \mathrm{~g}$ & $3 \mathrm{~h}$ & $3 \mathrm{i}$ & $3 \mathrm{j}$ & $3 \mathrm{k}$ & $9 \mathrm{a}$ & $9 \mathrm{~b}$ & $3 \mathrm{l}$ & $9 \mathrm{c}$ & $9 \mathrm{~d}$ \\
$\chi_{1}$ & 1 & 1 & 1 & 1 & 1 & 1 & 1 & 1 & 1 & 1 & 1 & 1 & 1 & 1 & 1 & 1 & 1 \\
$\chi_{2}$ & 1 & 1 & 1 & 1 & 1 & 1 & 1 & 1 & 1 & 1 & 1 & $A$ & $A$ & $A$ & $\bar{A}$ & $\bar{A}$ & $\bar{A}$ \\
$\chi_{3}$ & 1 & 1 & 1 & 1 & 1 & 1 & 1 & 1 & 1 & 1 & 1 & $\bar{A}$ & $\bar{A}$ & $\bar{A}$ & $A$ & $A$ & $A$ \\
$\chi_{4}$ & 1 & $A$ & $\bar{A}$ & $\bar{A}$ & 1 & 1 & $A$ & 1 & $A$ & $\bar{A}$ & 1 & 1 & $A$ & $\bar{A}$ & 1 & $A$ & $\bar{A}$ \\
$\chi_{5}$ & 1 & $\bar{A}$ & $A$ & $A$ & 1 & 1 & $\bar{A}$ & 1 & $\bar{A}$ & $A$ & 1 & 1 & $\bar{A}$ & $A$ & 1 & $\bar{A}$ & $A$ \\
$\chi_{6}$ & 1 & $A$ & $\bar{A}$ & $\bar{A}$ & 1 & 1 & $A$ & 1 & $A$ & $\bar{A}$ & 1 & $A$ & $\bar{A}$ & 1 & $\bar{A}$ & 1 & $A$ \\
$\chi_{7}$ & 1 & $\bar{A}$ & $A$ & $A$ & 1 & 1 & $\bar{A}$ & 1 & $\bar{A}$ & $A$ & 1 & $\bar{A}$ & $A$ & 1 & $A$ & 1 & $\bar{A}$ \\
$\chi_{8}$ & 1 & $A$ & $\bar{A}$ & $\bar{A}$ & 1 & 1 & $A$ & 1 & $A$ & $\bar{A}$ & 1 & $\bar{A}$ & 1 & $A$ & $A$ & $\bar{A}$ & 1 \\
$\chi_{9}$ & 1 & $\bar{A}$ & $A$ & $A$ & 1 & 1 & $\bar{A}$ & 1 & $\bar{A}$ & $A$ & 1 & $A$ & 1 & $\bar{A}$ & $\bar{A}$ & $A$ & 1 \\
$\chi_{10}$ & 3 & $B$ & $\bar{B}$ & $-C$ & 0 & 0 & $C$ & $\bar{D}$ & $\bar{B}$ & $-B$ & $D$ & 0 & 0 & 0 & 0 & 0 & 0 \\
$\chi_{11}$ & 3 & $\bar{B}$ & $B$ & $C$ & 0 & 0 & $-C$ & $D$ & $-B$ & $\bar{B}$ & $\bar{D}$ & 0 & 0 & 0 & 0 & 0 & 0 \\
\hline & & & & & & & & & & & & & & & & & \\
& & & & & & & & & & & & & & & & & \\
\end{tabular}




\section{Continued}

\begin{tabular}{cccccccccccccccccc}
\hline$\chi_{12}$ & 3 & $C$ & $-C$ & $-B$ & 0 & 0 & $-\bar{B}$ & $D$ & $B$ & $\bar{B}$ & $D$ & 0 & 0 & 0 & 0 & 0 & 0 \\
$\chi_{13}$ & 3 & $-C$ & $C$ & $-\bar{B}$ & 0 & 0 & $-B$ & $D$ & $\bar{B}$ & $B$ & $\bar{D}$ & 0 & 0 & 0 & 0 & 0 & 0 \\
$\chi_{14}$ & 3 & $-B$ & $-\bar{B}$ & $B$ & 0 & 0 & $\bar{B}$ & $D$ & $-C$ & $C$ & $\bar{D}$ & 0 & 0 & 0 & 0 & 0 & 0 \\
$\chi_{15}$ & 3 & $-\bar{B}$ & $-B$ & $\bar{B}$ & 0 & 0 & $B$ & $\bar{D}$ & $C$ & $-C$ & $D$ & 0 & 0 & 0 & 0 & 0 & 0 \\
$\chi_{16}$ & 3 & 0 & 0 & 0 & $D$ & $\bar{D}$ & 0 & 3 & 0 & 0 & 3 & 0 & 0 & 0 & 0 & 0 & 0 \\
$\chi_{17}$ & 3 & 0 & 0 & 0 & $\bar{D}$ & $D$ & 0 & 3 & 0 & 0 & 3 & 0 & 0 & 0 & 0 & 0 & 0 \\
\hline where: & $A=E(3)^{2}=\frac{-1-\operatorname{Sqrt}(-3)}{2} ;$ & $B=-E(3)-2 * E(3)^{2}=\frac{3+\operatorname{Sqrt}(-3)}{2} ;$ \\
$C=-E(3)+E(3)^{2}=-\operatorname{Sqrt}(-3) ;$ & $D=3 * E(3)^{2}=(-3-3 * \operatorname{Sqrt}(-3)) / 2$.
\end{tabular}

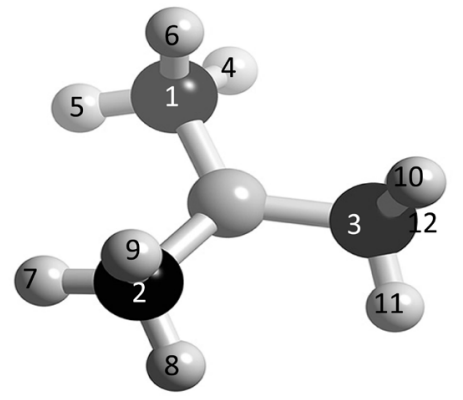

Figure 1. The structure of trimethylborane.

\section{2. f-NRG of Cyclohexane}

Consider the cyclohexane compound $\mathrm{C}_{6} \mathrm{H}_{12}$ with the structure.

The speediness of the rotation of the hexane group is considered suitably high that makes the mean time dynamical symmetry of the molecules makes sense. First considering the symmetry of $\mathrm{C}_{6}$ which is a cyclic group of order 6 namely $\mathbb{Z}_{6}$ denoted by $A:=\langle(1,2,3,4,5,6)\rangle$ (as shown in Figure 2). Also the remaining 6 corners of the framework are given by: $B_{1}:=\langle(7,8)\rangle, B_{2}:=\langle(9,10)\rangle$, $B_{3}:=\langle(11,12)\rangle, \quad B_{4}:=\langle(13,14)\rangle, \quad B_{5}:=\langle(15,16)\rangle$ and $B_{6}:=\langle(17,18)\rangle$ where $B_{i}$ is the symmetry of the $\mathrm{H}_{2}$ whose carbon atom is marked as $i, 1 \leq i \leq 6$. Therefore, the full symmetry of cyclohexane is:

$G=\left(B_{1} \times B_{2} \times B_{3} \times B_{4} \times B_{5} \times B_{6}\right) \rtimes A$. Which we can write in terms of wreath product as $G:=\mathbb{Z}_{2} w r \mathbb{Z}_{6}$. We used GAP package to get the group as follows:

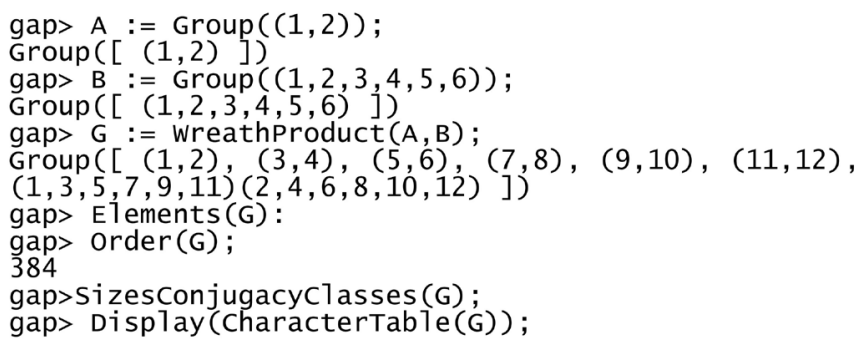

(See $[10])$ 
From the foregoing Table 3, The Representative of conjugacy classes of the group and Table 4, The Character table obtained gives room for the needed classification of wavefunctions, determination of selection rules, and so on.

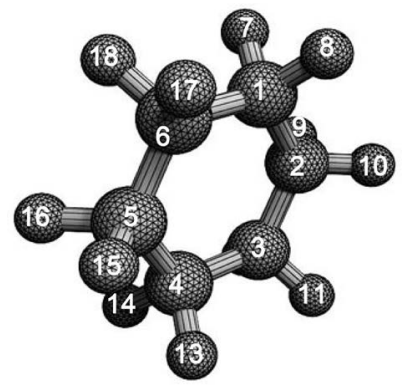

Figure 2. The structure of cyclohexane.

Table 3. The conjugacy class table for the group.

\begin{tabular}{|c|c|c|c|}
\hline $\mathrm{S} / \mathrm{N}$ & Representatives & Size & Name \\
\hline 1. & () & 1 & $1 \mathrm{a}$ \\
\hline 2. & $(11,12)$ & 6 & $2 \mathrm{a}$ \\
\hline 3. & $(9,10)(11,12)$ & 6 & $2 b$ \\
\hline 4. & $(7,8)(11,12)$ & 6 & $2 c$ \\
\hline 5. & $(7,8)(9,10)(11,12)$ & 6 & $2 \mathrm{~d}$ \\
\hline 6. & $(5,6)(11,12)$ & 6 & $2 \mathrm{e}$ \\
\hline 7. & $(5,6)(9,10)(11,12)$ & 6 & $2 \mathrm{f}$ \\
\hline 8. & $(5,6)(7,8)(11,12)$ & 6 & $2 \mathrm{~g}$ \\
\hline 9. & $(5,6)(7,8)(9,10)(11,12)$ & 6 & $2 \mathrm{~h}$ \\
\hline 10. & $(3,4)(7,8)(11,12)$ & 6 & $2 \mathrm{i}$ \\
\hline 11. & $(3,4)(7,8)(9,10)(11,12)$ & 6 & $2 \mathrm{j}$ \\
\hline 12. & $(3,4)(5,6)(9,10)(11,12)$ & 6 & $2 \mathrm{k}$ \\
\hline 13. & $(3,4)(5,6)(7,8)(9,10)(11,12)$ & 6 & 21 \\
\hline 14. & $(1,2)(3,4)(5,6)(7,8)(9,10)(11,12)$ & 1 & $2 \mathrm{~m}$ \\
\hline 15. & $(1,3,5,7,9,11)(2,4,6,8,10,12)$ & 32 & $6 f$ \\
\hline 16. & $(1,3,5,7,9,11,2,4,6,8,10,12)$ & 32 & $12 \mathrm{~b}$ \\
\hline 17. & $(1,5,9)(2,6,10)(3,7,11)(4,8,12)$ & 16 & $3 b$ \\
\hline 18. & $(1,5,9)(2,6,10)(3,7,11,4,8,12)$ & 32 & $6 \mathrm{~d}$ \\
\hline 19. & $(1,5,9,2,6,10)(3,7,11,4,8,12)$ & 32 & $6 e$ \\
\hline 20. & $(1,7)(2,8)(3,9)(4,10)(5,11)(6,12)$ & 8 & $2 n$ \\
\hline 21. & $(1,7)(2,8)(3,9)(4,10)(5,11,6,12)$ & 24 & $4 \mathrm{a}$ \\
\hline 22. & $(1,7)(2,8)(3,9,4,10)(5,11,6,12)$ & 24 & $4 \mathrm{~b}$ \\
\hline 23. & $(1,7,2,8)(3,9,4,10)(5,11,6,12)$ & 8 & $4 c$ \\
\hline 24. & $(1,9,5)(2,10,6)(3,11,7)(4,12,8)$ & 16 & $3 a$ \\
\hline 25. & $(1,9,5)(2,10,6)(3,11,8,4,12,7)$ & 32 & $6 \mathrm{~b}$ \\
\hline 26. & $(1,9,6,2,10,5)(3,11,8,4,12,7)$ & 16 & $6 c$ \\
\hline 27. & $(1,11,9,7,5,3)(2,12,10,8,6,4)$ & 32 & $6 a$ \\
\hline 28. & $(1,11,10,8,6,4,2,12,9,7,5,3)$ & 32 & $12 \mathrm{a}$ \\
\hline
\end{tabular}


Table 4. Character table for Cyclohexane molecule.

\begin{tabular}{|c|c|c|c|c|c|c|c|c|c|c|c|c|c|c|c|c|c|c|c|c|c|c|c|c|c|c|c|c|}
\hline & $1 \mathrm{a}$ & $2 \mathrm{a}$ & $2 b$ & $2 c$ & $2 \mathrm{~d}$ & $2 \mathrm{e}$ & $2 f$ & $2 \mathrm{~g}$ & $2 \mathrm{~h}$ & $2 \mathrm{i}$ & $2 j$ & $2 \mathrm{k}$ & 21 & $2 \mathrm{~m}$ & $6 a$ & $12 \mathrm{a}$ & $3 a$ & $6 \mathrm{~b}$ & $6 c$ & $2 \mathrm{n}$ & $4 \mathrm{a}$ & $4 \mathrm{~b}$ & $4 c$ & $3 b$ & $6 \mathrm{~d}$ & $6 e$ & $6 f$ & $12 \mathrm{~b}$ \\
\hline $3 \mathrm{P}$ & $1 \mathrm{a}$ & 1a & $1 \mathrm{a}$ & 1a & 1a & 1a & 1a & 1a & $1 \mathrm{a}$ & $1 \mathrm{a}$ & 1a & 1a & 1a & $1 \mathrm{a}$ & $3 a$ & $6 c$ & $3 b$ & $3 b$ & $3 b$ & $1 \mathrm{a}$ & $2 \mathrm{e}$ & $2 \mathrm{k} 2$ & $2 \mathrm{~m}$ & $3 a$ & $3 a$ & $3 a$ & $3 b$ & $6 e$ \\
\hline $3 \mathrm{P}$ & $1 \mathrm{a}$ & $2 \mathrm{a}$ & $2 \mathrm{~b}$ & $2 c$ & $2 \mathrm{~d}$ & $2 \mathrm{e}$ & $2 \mathrm{f}$ & $2 \mathrm{~g}$ & $2 \mathrm{~h}$ & $2 \mathrm{i}$ & $2 \mathrm{j}$ & $2 \mathrm{k}$ & 21 & $2 \mathrm{~m}$ & $2 n$ & $4 c$ & 1a & $2 \mathrm{i}$ & $2 \mathrm{~m}$ & $2 n$ & $4 \mathrm{a}$ & $4 \mathrm{~b}$ & $4 c$ & 1a & $2 \mathrm{i}$ & $2 \mathrm{~m}$ & $2 n$ & $4 c$ \\
\hline $5 \mathrm{P}$ & $1 \mathrm{a}$ & $2 a$ & $2 \mathrm{~b}$ & $2 c$ & $2 \mathrm{~d}$ & $2 \mathrm{e}$ & $2 \mathrm{f}$ & $2 \mathrm{~g}$ & $2 \mathrm{~h}$ & $2 \mathrm{i}$ & $2 \mathrm{j}$ & $2 \mathrm{k}$ & 21 & $2 \mathrm{~m}$ & $6 f$ & $12 \mathrm{~b}$ & $3 b$ & $6 \mathrm{~d}$ & $6 e$ & $2 n$ & $4 \mathrm{a}$ & $4 \mathrm{~b}$ & $4 c$ & $3 a$ & $6 \mathrm{~b}$ & $6 c$ & $6 a$ & $12 \mathrm{a}$ \\
\hline $7 \mathrm{P}$ & $1 \mathrm{a}$ & $2 a$ & $2 b$ & $2 c$ & $2 \mathrm{~d}$ & $2 \mathrm{e}$ & $2 \mathrm{f}$ & $2 \mathrm{~g}$ & $2 \mathrm{~h}$ & $2 \mathrm{i}$ & $2 j$ & $2 \mathrm{k}$ & 21 & $2 \mathrm{~m}$ & $6 a$ & $12 \mathrm{a}$ & $3 a$ & $6 \mathrm{~b}$ & $6 c$ & $2 \mathrm{n}$ & $4 \mathrm{a}$ & $4 \mathrm{~b}$ & $4 c$ & $3 b$ & $6 \mathrm{~d}$ & $6 e$ & $6 \mathrm{f}$ & $12 \mathrm{~b}$ \\
\hline $11 \mathrm{P}$ & $1 \mathrm{a}$ & $2 \mathrm{a}$ & $2 b$ & $2 c$ & $2 \mathrm{~d}$ & $2 \mathrm{e}$ & $2 \mathrm{f}$ & $2 \mathrm{~g}$ & $2 \mathrm{~h}$ & $2 \mathrm{i}$ & $2 j$ & $2 \mathrm{k}$ & 21 & $2 \mathrm{~m}$ & $6 f$ & $12 \mathrm{~b}$ & $3 b$ & $6 \mathrm{~d}$ & $6 e$ & $2 \mathrm{n}$ & $4 \mathrm{a}$ & $4 \mathrm{~b}$ & $4 c$ & $3 a$ & $6 \mathrm{~b}$ & $6 c$ & $6 a$ & $12 \mathrm{a}$ \\
\hline$\chi_{1}$ & 1 & 1 & 1 & 1 & 1 & 1 & 1 & 1 & 1 & 1 & 1 & 1 & 1 & 1 & 1 & 1 & 1 & 1 & 1 & 1 & 1 & 1 & 1 & 1 & 1 & 1 & 1 & 1 \\
\hline$\chi_{2}$ & 1 & -1 & 1 & 1 & -1 & 1 & -1 & -1 & 1 & 1 & -1 & 1 & -1 & 1 & 1 & -1 & 1 & -1 & 1 & -1 & 1 & -1 & 1 & 1 & -1 & 1 & -1 & 1 \\
\hline$\chi_{3}$ & 1 & -1 & 1 & 1 & -1 & 1 & -1 & -1 & 1 & -1 & 1 & 1 & -1 & 1 & 1 & -1 & 1 & -1 & 1 & -1 & 1 & 1 & -1 & 1 & -1 & 1 & 1 & -1 \\
\hline$\chi_{4}$ & 1 & 1 & 1 & 1 & 1 & 1 & 1 & 1 & 1 & 1 & 1 & 1 & 1 & 1 & -1 & -1 & 1 & 1 & 1 & -1 & -1 & -1 & -1 & 1 & 1 & 1 & -1 & -1 \\
\hline$\chi_{5}$ & 1 & -1 & 1 & 1 & -1 & 1 & -1 & -1 & 1 & -1 & 1 & 1 & -1 & 1 & $A$ & $-A$ & $-\bar{A}$ & $\bar{A}$ & $-\bar{A}$ & -1 & 1 & -1 & 1 & $-A$ & $A$ & $-A$ & $\bar{A}$ & $-\bar{A}$ \\
\hline$\chi_{6}$ & 1 & -1 & 1 & 1 & -1 & 1 & -1 & -1 & 11 & -1 & 1 & 1 & -1 & 1 & $\bar{A}$ & $-\bar{A}$ & $-A$ & $A$ & $-A$ & -1 & 1 & -1 & 1 & $-\bar{A}$ & $\bar{A}$ & $-\bar{A}$ & $A$ & $-A$ \\
\hline$\chi_{7}$ & 1 & -1 & 1 & 1 & -1 & 1 & -1 & -1 & 1 & -1 & 1 & 1 & -1 & 1 & $-\bar{A}$ & $\bar{A}$ & $-A$ & $A$ & $-A$ & 1 & -1 & 1 & -1 & $-\bar{A}$ & $\bar{A}$ & $-\bar{A}$ & $-A$ & $A$ \\
\hline$\chi_{8}$ & 1 & -1 & 1 & 1 & -1 & 1 & -1 & -1 & 1 & -1 & 1 & 1 & -1 & 1 & $-A$ & $A$ & $-\bar{A}$ & $\bar{A}$ & $-\bar{A}$ & 1 & -1 & 1 & -1 & $-A$ & $A$ & $-A$ & $-\bar{A}$ & $\bar{A}$ \\
\hline$\chi_{9}$ & 1 & 1 & 1 & 1 & 1 & 1 & 1 & 1 & 1 & 1 & 1 & 1 & 1 & 1 & $A$ & $A$ & $-\bar{A}$ & $-\bar{A}$ & $-\bar{A}$ & -1 & -1 & -1 & -1 & $-A$ & $-A$ & $-A$ & $\bar{A}$ & $\bar{A}$ \\
\hline$\chi_{10}$ & 1 & 1 & 1 & 1 & 1 & 1 & 1 & 1 & 1 & 1 & 1 & 1 & 1 & 1 & $\bar{A}$ & $\bar{A}$ & $-A$ & $-A$ & $-A$ & -1 & -1 & -1 & -1 & $-\bar{A}$ & $-\bar{A}$ & $-\bar{A}$ & $A$ & $A$ \\
\hline$\chi_{11}$ & 1 & 1 & 1 & 1 & 1 & 1 & 1 & 1 & 1 & 1 & 1 & 1 & 1 & 1 & $-\bar{A}$ & $-\bar{A}$ & $-A$ & $-A$ & $-A$ & 1 & 1 & 1 & 1 & $-\bar{A}$ & $-\bar{A}$ & $-\bar{A}$ & $-A$ & $-A$ \\
\hline$\chi_{12}$ & 1 & 1 & 1 & 1 & 1 & 1 & 1 & 1 & 1 & 1 & 1 & 1 & 1 & 1 & $-A$ & $-A$ & $-\bar{A}$ & $-\bar{A}$ & $-\bar{A}$ & 1 & 1 & 1 & 1 & $-A$ & $-A$ & $-A$ & $-\bar{A}$ & $-\bar{A}$ \\
\hline$\chi_{13}$ & 2 & 0 & -2 & 2 & 0 & -2 & 0 & 0 & 2 & 0 & -2 & 2 & 0 & -2 & 0 & 0 & 2 & 0 & -2 & 0 & 0 & 0 & 0 & 2 & 0 & -2 & 0 & 0 \\
\hline$\chi_{14}$ & 2 & 0 & -2 & 2 & 0 & -2 & 0 & 0 & 2 & 0 & -2 & 2 & 0 & -2 & 0 & 0 & $B$ & 0 & $-B$ & 0 & 0 & 0 & 0 & $\bar{B}$ & 0 & $-\bar{B}$ & 0 & 0 \\
\hline$\chi_{15}$ & 2 & 0 & -2 & 2 & 0 & -2 & 0 & 0 & 2 & 0 & -2 & 2 & 0 & -2 & 0 & 0 & $\bar{B}$ & 0 & $-\bar{B}$ & 0 & 0 & 0 & 0 & $B$ & 0 & $-B$ & 0 & 0 \\
\hline$\chi_{16}$ & 3 & -1 & -1 & -1 & 3 & 3 & -1 & -1 & -1 & 3 & -1 & 3 & -1 & 3 & 0 & 0 & 0 & 0 & 0 & -3 & 1 & 1 & -3 & 0 & 0 & 0 & 0 & 0 \\
\hline$\chi_{17}$ & 3 & -1 & -1 & -1 & 3 & 3 & -1 & -1 & -1 & 3 & -1 & 3 & -1 & 3 & 0 & 0 & 0 & 0 & 0 & 3 & -1 & -1 & 3 & 0 & 0 & 0 & 0 & 0 \\
\hline$\chi_{18}$ & 3 & 1 & -1 & -1 & -3 & 3 & 1 & 1 & -1 & -3 & -1 & 3 & 1 & 3 & 0 & 0 & 0 & 0 & 0 & -3 & -1 & 1 & 3 & 0 & 0 & 0 & 0 & 0 \\
\hline$\chi_{19}$ & 3 & 1 & -1 & -1 & -3 & 3 & 1 & 1 & -1 & -3 & -1 & 3 & 1 & 3 & 0 & 0 & 0 & 0 & 0 & 3 & 1 & -1 & -3 & 0 & 0 & 0 & 0 & 0 \\
\hline$\chi_{20}$ & 6 & -2 & -2 & 2 & 2 & -2 & 2 & 2 & -2 & -6 & 2 & -2 & -2 & 6 & 0 & 0 & 0 & 0 & 0 & 0 & 0 & 0 & 0 & 0 & 0 & 0 & 0 & 0 \\
\hline$\chi_{21}$ & 6 & -2 & 2 & -2 & -2 & -2 & 2 & 2 & 2 & 6 & -2 & -2 & -2 & 6 & 0 & 0 & 0 & 0 & 0 & 0 & 0 & 0 & 0 & 0 & 0 & 0 & 0 & 0 \\
\hline$\chi_{22}$ & 6 & 2 & -2 & 2 & -2 & -2 & -2 & -2 & -2 & 6 & 2 & -2 & 2 & 6 & 0 & 0 & 0 & 0 & 0 & 0 & 0 & 0 & 0 & 0 & 0 & 0 & 0 & 0 \\
\hline$\chi_{23}$ & 6 & 2 & 2 & -2 & 2 & -2 & -2 & -2 & 2 & -6 & -2 & -2 & 2 & 6 & 0 & 0 & 0 & 0 & 0 & 0 & 0 & 0 & 0 & 0 & 0 & 0 & 0 & 0 \\
\hline$\chi_{24}$ & 6 & 0 & 2 & -2 & 0 & -6 & 0 & 0 & -2 & 0 & 2 & 6 & 0 & -6 & 0 & 0 & 0 & 0 & 0 & 0 & 0 & 0 & 0 & 0 & 0 & 0 & 0 & 0 \\
\hline$\chi_{25}$ & 6 & -4 & 2 & 2 & 0 & 2 & 0 & 0 & -2 & 0 & -2 & -2 & 4 & -6 & 0 & 0 & 0 & 0 & 0 & 0 & 0 & 0 & 0 & 0 & 0 & 0 & 0 & 0 \\
\hline$\chi_{26}$ & 6 & 0 & -2 & -2 & 0 & 2 & -4 & 4 & 2 & 0 & 2 & -2 & 0 & -6 & 0 & 0 & 0 & 0 & 0 & 0 & 0 & 0 & 0 & 0 & 0 & 0 & 0 & 0 \\
\hline$\chi_{27}$ & 6 & 0 & -2 & -2 & 0 & 2 & 4 & -4 & 2 & 0 & 2 & -2 & 0 & -6 & 0 & 0 & 0 & 0 & 0 & 0 & 0 & 0 & 0 & 0 & 0 & 0 & 0 & 0 \\
\hline$\chi_{28}$ & 6 & 4 & 2 & 2 & 0 & 2 & 0 & 0 & -2 & 0 & -2 & -2 & -4 & -6 & 0 & 0 & 0 & 0 & 0 & 0 & 0 & 0 & 0 & 0 & 0 & 0 & 0 & 0 \\
\hline
\end{tabular}

where: $A=-E(3)=\frac{1-\operatorname{Sqrt}(-3)}{2} ; \quad B=2 * E(3)=-1+\operatorname{Sqrt}(-3)$. 


\section{Conclusion}

In this paper, we computed the group, the conjugacy classes and character tables of Trimethylborane and Cyclohaxane as seen in Tables 1-4. We found that the groups are isomorphic to the wreath products $C_{3} w r C_{2}$ and $C_{2} w r C_{6}$ with orders 81 and 384 and with 17 and 28 conjugacy classes respectively, where $C_{n}$ is cyclic group of order $n$. We used the GAP package for our calculations. The character tables obtained give room for the needed classification of wavefunctions, determination of selection rules, and so on.

\section{Conflicts of Interest}

The authors declare no conflicts of interest regarding the publication of this paper.

\section{References}

[1] Balasubramanian, K. (1984) Recent Applications of Group Theoretical Generators to Chemical Physics. Croatica Chemica Acta, 57, 1525-1552.

[2] Hamadanian, M. and Ashrafi, A.R. (2003) The Full Non-Rigid Group Theory for Trimethylamine. IJMMS, 42, 2701-2706. https://doi.org/10.1155/S0161171203205159

[3] Darafsher, M.R., Ashrafi, A.R. and Darasher, A. (2005) Group Theory for Tetramethylethylene. Acta Chimica Slovenica, 52, 282-287.

[4] Darafsher, M.R., Farjami, Y. and Ashrafi, A.R. (2005) Computing the Full Non-Rigid Group of Tetranitrocubane and Octanitrocubunae Using Wreath Product. Communications in Mathmatical and in Computer Chemistry, 54, 53-74.

[5] Darafsher, M.R., Ashrafi, A.R. and Darafsher, A. (2007) Non-Rigid Group Theory for 2,3-Dimethybutane. MATCH Communications in Mathematical and in Computer Chemistry, 58, 47-56.

[6] Moghani, A., Sedeh, S.N. and Sorouhesh, M.R. (2010) The Fujita Combinatorial Enumeration for the Non-Rigid Group of 2,4-Dimethylbenzene. Journal of the Serbian Chemical Society, 75, 91-99. https://doi.org/10.2298/JSC1001091M

[7] Moghadam, M.E., Karimi, T. and Algar, S. (2012) Group Theory for Non-Rigid Methyl Borane Derivatives. 22nd Iranian Algebra Seminar, Hakim Sabzevari University, Iran, 314-317.

[8] Moghadam, M.E., Karimi, T. and Farrokhi, M.D. (2012) Full Non-Rigid Group Theory and Symmetry of Some Diborane Derivatives. International Journal of Physical Sciences, 7, 73-80. https://doi.org/10.5897/IJPS11.1646

[9] Dixon, J.D. and Mortimer, B. (1996) Permutation Groups. Springer-Verlag, Berlin, New York. https://doi.org/10.1007/978-1-4612-0731-3

[10] The GAP Group (2018) GAP-Reference Manual, Release 4.8.10. 23 June 2019. https://www.gap-system.org 